\title{
Pitfall of the Detection Rate Optimized Bit Allocation within Template Protection and a Remedy
}

\author{
E.J.C. Kelkboom, K.T.J. de Groot, C. Chen, J. Breebaart, R.N.J. Veldhuis
}

\begin{abstract}
One of the requirements of a biometric template protection system is that the protected template ideally should not leak any information about the biometric sample or its derivatives. In the literature, several proposed template protection techniques are based on binary vectors. Hence, they require the extraction of a binary representation from the real- valued biometric sample. In this work we focus on the Detection Rate Optimized Bit Allocation (DROBA) quantization scheme that extracts multiple bits per feature component while maximizing the overall detection rate. The allocation strategy has to be stored as auxiliary data for reuse in the verification phase and is considered as public. This implies that the auxiliary data should not leak any information about the extracted binary representation. Experiments in our work show that the original DROBA algorithm, as known in the literature, creates auxiliary data that leaks a significant amount of information. We show how an adversary is able to exploit this information and significantly increase its success rate on obtaining a false accept. Fortunately, the information leakage can be mitigated by restricting the allocation freedom of the DROBA algorithm. We propose a method based on population statistics and empirically illustrate its effectiveness. All the experiments are based on the MCYT fingerprint database using two different texture based feature extraction algorithms.
\end{abstract}

\section{INTRODUCTION}

The widespread use of biometric systems introduces new privacy risks, for example identity theft or cross-matching. These risks can be mitigated by applying template protection techniques. An overview of the privacy risks and template protection techniques are presented in [1]. A subclass of template protection techniques is based on a transformation of a biometric measurement to a binary vector as initial step. Hence, they require the extraction of a binary representation from the real- valued biometric sample. In the literature, numerous quantization schemes have been proposed. They vary from a simple method of extracting a single bit per feature component [2][3] to a more complex, multiple bits per feature component, extraction method [4][5][6][7]. If the quantization scheme is subject-specific the information has to be stored as auxiliary data for further use in the verification phase.

One of the requirements of a template protection system is that the stored auxiliary data ideally should not leak any information about the binary representation or the biometric sample itself. Hence, the subject-specific quantization

E.J.C. Kelkboom, K.T.J. de Groot, and J. Breebaart are with Philips Research, The Netherlands $\{$ Emile.Kelkboom, Koen.de.Groot, Jeroen. Breebaart $\}$ aphilips.com

C. Chen and R.N.J. Veldhuis are with the University of Twente, Fac. EEMCS, The Netherlands $\{\mathrm{C}$. Chen, R.N.J.Veldhuis\}autwente.nl scheme stored as the auxiliary data should not reveal any information that may facilitate an adversary on increasing its success rate guessing the binary representation of the biometric sample in order to obtain a false accept.

The work of [8] showed that the quantization schemes proposed in [9] and [10] do indeed leak information that could be exploited by an adversary. Their attack model is to guess the secret key in an off-line mode by using the auxiliary data and population statistics. They use the guessing distance, consisting of the number of attempts required for a correct guess, as the measure of the degree of difficulty. Their results showed that the guessing distance is much smaller than what is expected based on the claimed security in [9] and [10], respectively. We focus on the Detection Rate Optimized Bit Allocation (DROBA) quantization scheme proposed in [7] that extracts multiple bits per feature component. For each enrolled subject the optimization algorithm allocates the optimal number of bits per component while maximizing the overall detection rate. The bit allocation strategy has to be stored as auxiliary data for further use during the verification phase.

Contribution: Our contribution is threefold. Firstly, we show that if the DROBA quantization scheme is not correctly implemented it will leak information about the binary representation of the biometric sample. Secondly, we illustrate an attack method an adversary could use in order to increase its success rate on reproducing a binary representation that leads to a false accept. Instead of using the guessing distance, we use the false-acceptance rate $(\mathrm{FAR}, \alpha)$ as the degree of difficulty. We consider the template protection technique known as the helperdata system [2][3][11]. However, any template protection technique incorporating the DROBA quantization scheme is susceptible to this vulnerability. Thirdly, we outline a solution and propose an implementation guideline as a remedy. The remedy significantly mitigates the information leakage and guarantees a more private template.

The outline of this paper is as follows. In Section II we briefly discuss the considered template protection system with the DROBA quantization scheme. In Section III we describe our experimental setup concerning a fingerprint database, two feature extraction algorithms, and a testing protocol followed by the analysis of the information leakage due to the improper implementation of the DROBA quantization scheme. With use of the information leakage we demonstrate an attack method in Section IV that significantly increases 


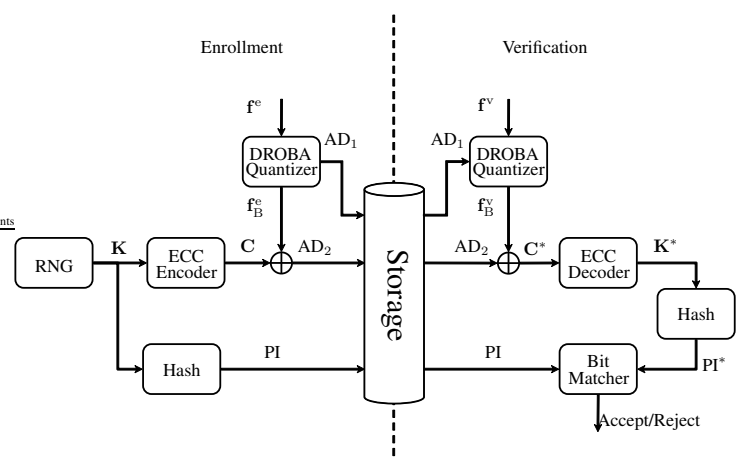

Fig. 1. Template protection scheme with DROBA implementation.

the false accept probability. As a remedy, we propose an implementation guideline in Section $\mathrm{V}$ and show that it significantly mitigates the information leakage. We finish with the conclusions in Section VI.

\section{Template Protection Scheme With DROBA}

The template protection technique under consideration is known as the helper-data system [2][3] [11] and is portrayed in Fig. 1. As input we have the real-valued feature vector of dimension $N_{\mathrm{F}}$, $\mathbf{f} \in \mathbb{R}^{N_{\mathrm{F}}}$, which is extracted from the biometric sample by the feature extraction algorithm. Subsequently, a binary vector $\mathbf{f}_{\mathrm{B}} \in\{0,1\}^{N_{\mathrm{B}}}$ is extracted by the DROBA quantization module and outputs the first auxiliary data $\mathrm{AD}_{1}$ containing the allocation strategy. Many template protection schemes are based on the capability of generating a robust binary vector or key out of different biometric measurements of the same subject. However, the binary vector $f_{B}$ itself cannot be used as the key because it is most likely not exactly the same in both the enrollment and verification phase $\left(\mathbf{f}_{\mathrm{B}}^{\mathrm{e}} \neq \mathbf{f}_{\mathrm{B}}^{\mathrm{v}}\right.$ ), due to measurement noise and biometric variability that lead to bit errors. The number of bit errors between two binary vectors is also referred to as the Hamming distance (HD) $d_{\mathrm{H}}\left(\mathbf{f}_{\mathrm{B}}^{\mathrm{e}}, \mathbf{f}_{\mathrm{B}}^{\mathrm{v}}\right)$. Therefore, ECCs are used to deal with these bit errors. As shown in Fig. 1, the ECC and hash function are integrated using the well-known Fuzzy Commitment scheme [12]. For the sake of coherence we use the terminology proposed in [13].

Within the fuzzy commitment scheme we use the linear block type ECC "Bose, Ray-Chaudhuri, Hocquenghem" $(\mathrm{BCH})$ that corrects random errors. The codeword $\mathbf{C}$ corresponding to a randomly generated secret $\mathbf{K}$ is XOR-ed with the $f_{B}^{e}$ in order to obtain the auxiliary data $A_{2}$. Furthermore, the hash of $\mathbf{K}$ is taken in order to obtain the pseudo identity PI. In the verification phase this process is reversed with help of the auxiliary data resulting into a candidate pseudo identity PI*. Only when $d_{\mathrm{H}}\left(\mathbf{f}_{\mathrm{B}}^{\mathrm{e}}, \mathbf{f}_{\mathrm{B}}^{\mathrm{v}}\right) \leq t_{\mathrm{c}}$ then PI and PI* are equal, thus resulting into an accept. Hence, the Fuzzy Commitment scheme can be considered as a HD-classifier. More details about the template protection system can be found in [2][3].

As mentioned previously, the binary vector $\mathbf{f}_{\mathrm{B}}$ is extracted from the real-valued input vector $\mathbf{f}$ by the DROBA quantization scheme and algorithm proposed in [7]. The DROBA algorithm has the flexibility to extract multiple bits from a single component. The number of bits extracted from component $i$ is given by $b_{i}$. The quantization schemes for the $b_{i} \in\{1,2,3\}$ cases are shown in Fig. 2(a), (b), and (c), respectively. For convenience we refer the $b_{i}=1$ case as $b_{1}^{*}$, and $b_{2}^{*}$ and $b_{3}^{*}$ for the $b_{i}=2$ and $b_{i}=3$ cases, respectively. The $2^{b_{i}}$ quantization intervals are defined as such that the occurrence of each interval is equiprobable with respect to the total density, which we assume to be Gaussian distributed $p_{\mathrm{t}} \sim \mathcal{N}\left(\mu_{\mathrm{t}}, \sigma_{\mathrm{t}}^{2}\right)$ with mean $\mu_{\mathrm{t}}$ and variance $\sigma_{\mathrm{t}}^{2}$. The total density defines the observed variability of that component across the whole population. Each quantization interval is assigned a unique $b_{i}$ bits Gray code [14]. Furthermore, we model the observed biometric variability and measurement errors of the feature vector component of a specific subject with the within-class density, which for simplicity is assumed to be another Gaussian density $p_{\mathrm{w}} \sim \mathcal{N}\left(\mu_{\mathrm{w}}, \sigma_{\mathrm{w}}^{2}\right)$. Note that $\mu_{\mathrm{w}}$ and $\sigma_{\mathrm{w}}^{2}$ can be different for each component or subject. From [7] the detection rate $\gamma$ is defined as the probability that the next measurement of the feature component will be in the same quantization interval. For component $i$ the detection rate is computed as

$$
\gamma_{i}\left(b_{i}\right)=\int_{Q_{\mu_{\mathrm{w}}}\left(b_{i}\right)} p_{\mathrm{w}}(v) \mathrm{d} v,
$$

where $Q_{\mu_{\mathrm{w}}}\left(b_{i}\right)$ is the quantization interval corresponding to $\mu_{\mathrm{w}}$ and also depends on the number of bits $b_{i}$ to be extracted. Thus, the detection rate is the part of the withinclass density within the quantization interval corresponding to $\mu_{\mathrm{w}}$, portrayed by the shaded area in Fig. 2. For the case where no bits are extracted $\left(b_{i}=0\right)$ the detection rate is defined as $\gamma_{i}(0)=1$. Note that the detection rate decreases when $b_{i}$ increases. Under the assumption that the $N_{\mathrm{F}}$ feature components are independent, the overall detection rate is defined as

$$
\gamma_{t}=\prod_{i=1}^{N_{\mathrm{F}}} \gamma_{i}\left(b_{i}\right)
$$

The DROBA algorithm has to create a binary vector of length $N_{\mathrm{B}}$, hence it has to allocate $N_{\mathrm{B}}$ bits across all components. We also refer to $N_{\mathrm{B}}$ as the bit-budget. With use of the multiple $\left(N_{\mathrm{e}}\right)$ enrollment samples, the DROBA algorithm analyzes the subject-dependent feature statistics $\left(\mu_{\mathrm{w}}\right.$ and $\left.\sigma_{\mathrm{w}}^{2}\right)$ of each component and allocates the optimal number of bits $b_{i}$ to component $i$ with the constrains of maximizing the overall detection rate $\gamma_{t}$ and allocating the bit-budget $\sum_{i=1}^{N_{\mathrm{F}}} b_{i}=N_{\mathrm{B}}$. The optimal allocation strategy is stored as auxiliary data $\mathrm{AD}_{1}=\left[b_{1}, b_{2}, \ldots, b_{N_{\mathrm{F}}}\right]$ for reuse at the verification phase. The optimization is implemented using the dynamic programming approach presented in [7].

\section{EXPERIMENTS}

If the DROBA implementation is correct, auxiliary data $\mathrm{AD}_{1}$ should not leak any information about the enrolled binary vector $\mathrm{f}_{\mathrm{B}}^{\mathrm{e}}$. We will empirically analyze whether there is any information leakage by means of a fingerprint database and two feature extraction algorithms. We first discuss the 


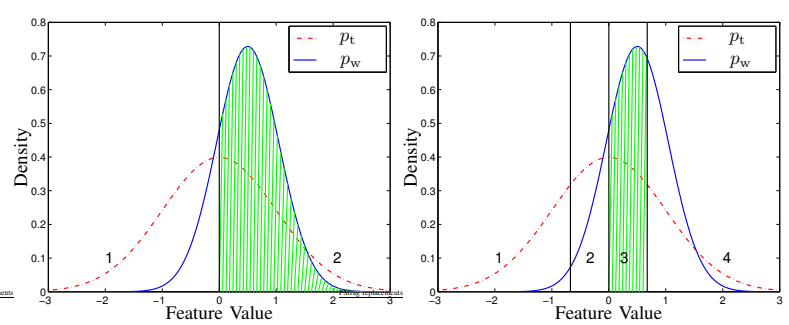

(a) $b_{1}^{*}$ case with 2 intervals

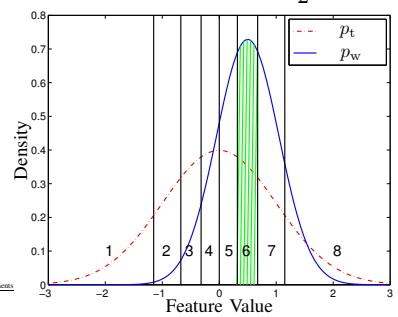

(c) $b_{3}^{*}$ case with 8 intervals

Fig. 2. The total density $p_{\mathrm{t}}$ with an example of a within-class density $p_{\mathrm{w}}$ and the corresponding detection rate $\gamma_{i}$ at different quantization scheme where (a) $b_{i}=1\left(b_{1}^{*}\right)$, (b) $b_{i}=2\left(b_{2}^{*}\right)$, (c) $b_{i}=3\left(b_{3}^{*}\right)$ bits are extracted.

experiment setup including the testing protocol followed by the information leakage analysis.

\section{A. Experiment Setup}

1) Biometric Modality and Database: The database we use is the MCYT (Ministerio de Ciencia y Tecnología) containing fingerprint images [15]. It contains 12 images of all 10 fingers from $N_{\mathrm{s}}=330$ subjects. However, we limit our dataset to the images of the right-index finger only.

2) Feature Extraction Algorithms: Two types of texture based features are extracted from a fingerprint, namely directional field and Gabor features. In order to compensate for possible translations between enrolled and verification measurements, a translation-only pre-alignment step is performed during the feature extraction process. Such pre-alignment requires extraction of the core point which is performed according to the algorithm described in [16]. Around the core point we define a $17 \times 17$ grid with eight pixels between each grid point. The following feature extraction algorithms extract a feature value on each grid point.

The first feature extraction algorithm is based on directional fields. A directional field vector describes the estimated local ridge-valley edge orientation in a fingerprint structure and is based on gradient vectors. The orientation of the ridgevalley edge is orthogonal to the gradient's angle. Therefore a directional field vector that signifies the orientation of the ridge-valley edge is perpendicular positioned to the gradient vector. In order to extract directional field features from a fingerprint the algorithm described in [17] is applied on each grid point. The direction field features have a dimension of $N_{\mathrm{F}}=578$ and are referred to as the DF features.

The second type of extracted features are the Gabor (GF) features, described in [18], where each grid point is filtered using a set of four 2D Gabor filters at angles of $\left\{0, \frac{\pi}{4}, \frac{\pi}{2}, \frac{3 \pi}{4}\right\}$. The feature vector is the concatenation of the modulus of the four complex responses at each grid point, resulting into a feature vector dimension of $N_{\mathrm{F}}=1156$.

3) Testing Protocol: The performance testing protocol consists of randomly selecting 220 out of $N_{\mathrm{s}}$ subjects as the training set and the remaining 110 subjects as the evaluation set, which is referred to as the training-evaluation-set split. To decorrelate the feature components we use the principle component analysis (PCA) and the linear discriminant analysis (LDA) techniques, where the LDA transformation is also used to obtain more discriminating feature components from which we expect to extract more bits from. The PCA and LDA transformation matrices are computed using this training set, where $N_{\mathrm{PCA}}$ is the reduced dimension after applying the PCA transformation and $N_{\mathrm{LDA}}$ is the reduced dimension after applying the LDA transformation. To avoid singularities we ensure that $N_{\mathrm{LDA}} \leq 220$. Furthermore, the template protection system parameters such as the quantization thresholds, used within the Bit Extraction module, are also estimated on the training set. From the evaluation set, 6 samples of each subject are randomly selected as the enrollment samples while the remaining samples are considered as the verification samples. This split is referred to as the enrollment-verification split. The protected template is generated using all the enrollment samples and compared with each individual verification sample. When the verification sample is from the same subject as of the protected template, it is referred to as a genuine comparison, otherwise it is an imposter comparison.

The training-evaluation-set split is performed five times, while for each of these splits the enrollment-verification split is performed 3 times. From each enrollment-verification split we estimate the $\beta_{\mathrm{tar}}$ (the false-rejection rate $(\mathrm{FRR}, \beta$ ) at the targeted FAR of $\alpha_{\mathrm{tar}}=0.1 \%$ ) and the equal-error rate (EER) where the FAR is equal to the FRR. Note, that the splits are performed randomly, however the seed at the start of the protocol is always the same, hence all the splits are equal for the performance tests at different settings. Hence, the splitting process does not contribute to any performance differences.

\section{B. Analysis of the Information Leakage}

First of all we empirically derive the $\left\{N_{\mathrm{PCA}}, N_{\mathrm{LDA}}, N_{\mathrm{B}}\right\}$ setting leading to the optimal performance in terms of $\beta_{\mathrm{tar}}$. We evaluate the performance for the settings of $N_{\mathrm{PCA}} \in\{50,100, \ldots, 300\}$ and $N_{\mathrm{B}} \in$ $\left\{50,100, \ldots, \min \left(N_{\mathrm{PCA}} \cdot b_{\max }, 300\right)\right\}$, while the $N_{\mathrm{LDA}}$ parameter is set to $N_{\mathrm{LDA}}=\min \left(N_{\mathrm{PCA}}, 220\right)$ as discussed in Section III-A.3. The achieved $\beta_{\text {tar }}$ performance for the different $\left\{N_{\mathrm{PCA}}, N_{\mathrm{LDA}}, N_{\mathrm{B}}\right\}$ settings are depicted in Fig 3(a) and (b) for the DF and GF features, respectively.

For the DF features the optimal setting is achieved at $\{150,150,100\}$, while at $\{200,200,100\}$ for the GF features. At the optimal performance settings, the error-rate ( $\alpha$ and $\beta$ ) curves with respect to the relative Hamming distance 


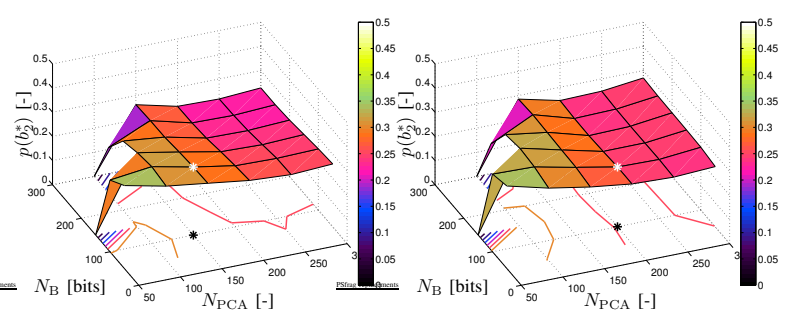

(a) DF: $p\left(b_{2}^{*}\right)$

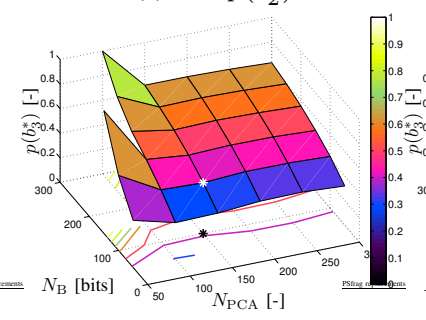

(c) DF: $p\left(b_{3}^{*}\right)$ (b) GF: $p\left(b_{2}^{*}\right)$

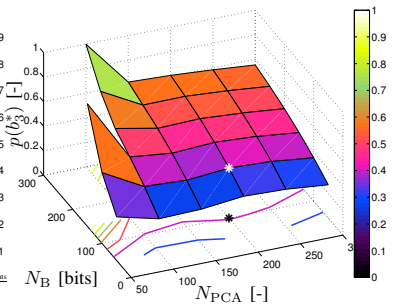

(d) GF: $p\left(b_{3}^{*}\right)$

Fig. 6. The $p\left(b_{2}^{*}\right), p\left(b_{3}^{*}\right)$ for different settings of $N_{\mathrm{PCA}}$ and $N_{\mathrm{B}}$ for the $\mathrm{DF}$ and GF features. The optimal performance setting is indicated with both the black and white star.

figures show that if $N_{\mathrm{B}}$ increases, more bits are extracted from the $b_{3}^{*}$ case and less from the $b_{1}^{*}$ case. The number of bits extracted from the $b_{2}^{*}$ case stays relatively stable. For the optimal setting we have the probabilities $p\left(b_{1}^{*}\right)=0.345$, $p\left(b_{2}^{*}\right)=0.247$, and $p\left(b_{3}^{*}\right)=0.408$ for the DF features, and $p\left(b_{1}^{*}\right)=0.304, p\left(b_{2}^{*}\right)=0.282$, and $p\left(b_{3}^{*}\right)=0.414$ for the GF features, respectively. Note that the majority of the bits are extracted from a multiple-bits extraction case, from which we know that information is leaked as shown in Fig. 5. More precisely, the largest portion of bits are extracted from the $b_{3}^{*}$ case, which leaks the most information.

\section{Exploitation of the Leakage}

In the previous section we have shown that the information leakage from the auxiliary data $\mathrm{AD}_{1}$ about the enrolled binary vector $\mathrm{f}_{\mathrm{B}}^{\mathrm{e}}$ is significant even at the optimal performance setting. However, it does not show what the actual practical advantage is for the adversary. In this section we propose a simple method the adversary could use in order to take advantage of the leaked information.

We consider the attack scenario where the adversary has the protected template, which is the collection of public auxiliary data $\mathrm{AD}_{1}, \mathrm{AD}_{2}$ and $\mathrm{PI}$, of an unknown subject and tries to obtain a false accept by the biometric system. As defined in [19] we focus on the attack level of "overriding the feature extraction process". A possible attack method would be a dictionary attack, where a random image sample from a publicly available fingerprint database is selected, its feature vector $\mathbf{f}$ is extracted and send to the next modules as

TABLE I

THE $p\left(b_{1}^{*}\right), p\left(b_{2}^{*}\right), p\left(b_{3}^{*}\right), \delta_{2}, \delta_{3}$ VALUES FOR THE DF AND GF FEATURES

\begin{tabular}{|c|c|c|c|c|c|c|c|}
\hline Features & EER [\%] & $\beta_{\text {tar }}[\%]$ & $p\left(b_{1}^{*}\right)$ & $p\left(b_{2}^{*}\right)$ & $p\left(b_{3}^{*}\right)$ & $\delta_{2}$ & $\delta_{3}$ \\
\hline \hline DF & 1.49 & 3.66 & 0.345 & 0.247 & 0.408 & 0.1706 & 0.4106 \\
GF & 1.29 & 2.30 & 0.304 & 0.282 & 0.414 & 0.3136 & 0.4727 \\
\hline
\end{tabular}

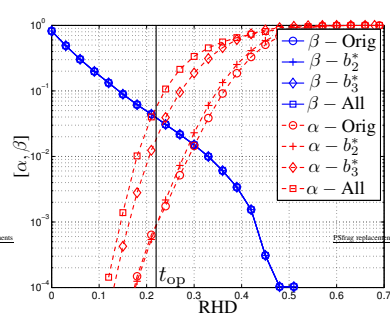

(a) DF: $\alpha$ and $\beta$ curves

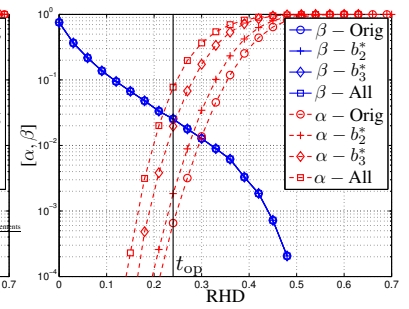

(b) GF: $\alpha$ and $\beta$ curves
Fig. 7. The error-rate curves pmfs for the (a) DF and (b) GF features when using the proposed attack at the imposter comparisons.

if it is authentic. The probability of an accept is equal to the FAR of the template protection system, because the imposter comparisons in fact do represent a dictionary attack. In our work, the targeted FAR is $\alpha_{\text {tar }}=0.1 \%$, thus on average $\frac{1}{\alpha_{\mathrm{tar}}}=1000$ attempts are expected in order to obtain a successful accept.

In our proposed attack method we also consider the DROBA Quantizer module to be compromised. Hence, the binary vector $\mathrm{f}_{\mathrm{B}}^{\mathrm{e}}$ is generated and send to the next module. The leaked information can be exploited in the following way. We change the DROBA Quantizer module as such that if multiple bits are extracted (the $b_{2}^{*}$ and $b_{3}^{*}$ cases indicated by $\mathrm{AD}_{1}$ ), we randomly select one of the two outer quantization intervals and return the corresponding Gray code. Hence, if $\mathrm{AD}_{1}$ indicates that it is a $b_{2}^{*}$ case, then either quantization intervals 1 or 4 are selected with $50 \%$ probability and when it is a $b_{3}^{*}$ case the quantization intervals 1 or 8 are selected at random.

The attack results are given by the error-rate curves in Fig. 7(a) and (b) for the DF and GF features, respectively. Note that the attack is only carried out on the imposter comparisons and hence only the FAR curves are influenced. The original FAR is indicated with the "Orig" suffix, which is previously shown in Fig. 4 and represents the case where the attacker plainly selects a random sample from the database for the verification comparison without using any available knowledge and is the common FAR reported in the literature. For the attacks including the knowledge of the information leakage, we first study the method where only the information leakage from the $b_{2}^{*}$ cases are exploited, hereafter we consider the method where only the $b_{3}^{*}$ cases are exploited, and as the last method both the $b_{2}^{*}$ and $b_{3}^{*}$ cases are exploited. These attack methods are indicated with the suffix " $b_{2}^{*}$ ", " $b_{3}^{*}$ ", and "All", respectively.

The operating point of a biometric system is determined using the $\alpha$-Orig curve. The closest operating point $t_{o p}$ where the FAR reaches the targeted $\alpha_{\mathrm{tar}}=0.1 \%$ without exceeding

TABLE II

THE OPERATING POINT $t_{o p}$ AT $\alpha_{\text {tar }}$ OF THE ORIGINAL CASE AND THE FAR OBTAINED AT THE DIFFERENT ATTACK SCENARIO.

\begin{tabular}{|c|cc|c|c|c|}
\hline & \multicolumn{2}{|c|}{ Orig case } & \multicolumn{3}{|c|}{ FAR at $t_{o p}$ at attack scenario } \\
\hline Features & $t_{o p}[\mathrm{RHD}]$ & $\approx \alpha_{\text {tar }}[\%]$ & $b_{2}^{*}[\%]$ & $b_{3}^{*}[\%]$ & All [\%] \\
\hline \hline $\mathrm{DF}$ & 0.22 & $8.71 \cdot 10^{-2}$ & $8.23 \cdot 10^{-2}$ & 1.89 & 5.78 \\
$\mathrm{GF}$ & 0.23 & $6.56 \cdot 10^{-2}$ & $1.84 \cdot 10^{-1}$ & 1.97 & 7.75 \\
\hline
\end{tabular}


it, is portrayed with the solid vertical line. The operating point is at a RHD $=0.22$ with $\alpha=8.71 \cdot 10^{-2} \%$ for the DF features and RHD $=0.23$ with $\alpha=6.56 \cdot 10^{-2} \%$ for the GF features. The FAR obtained at the operating point for the different attack methods are given in Table II. The results show that $\alpha-b_{3}^{*}$ is larger than $\alpha-b_{2}^{*}$, which confirms the fact that the information leakage of the $b_{3}^{*}$ cases is significantly larger than of the $b_{2}^{*}$ cases. Furthermore, the advantage of the adversary is further increased by using the information leakage of both cases, because $\alpha$-All is even larger. Hence, the largest achieved $\alpha$ is $5.78 \%$ for the DF features and $7.75 \%$ for the GF features. For the DF features the FAR has increased with a gain factor $G_{\alpha}=66$, while for the GF features $G_{\alpha}=118$. Thus, for both features the adversary gain is around two orders of magnitude. The necessary effort for the adversary to obtain an accept has significantly decreased from on average 1148 attempts to 17 attempts for the DF features and from 1524 to 13 for the GF features. Hence, the gain factor $G_{\alpha}$ can be seen as the gain of the adversary by exploiting the information leakage.

\section{An Implementation GUideline as REMEDY}

In the previous section we have shown that if no precaution is taken, an adversary with knowledge of the DROBA implementation could significantly increase its false-acceptance rate with two orders of magnitude by exploiting the information leakage embedded in the auxiliary data $\mathrm{AD}_{1}$ of the protected template. In this section we will address the cause of the information leakage and propose an implementation guideline for mitigating the leakage.

\section{A. The Cause}

Recall the fact that the DROBA algorithm is allowed to extract multiple bits from all feature components of $\mathbf{f}$, irrespective of its discriminating power or quality. Using the Gaussian model for describing the feature distribution of $\mathbf{f}$ (see Section II), we can analyze the detection rate at different subject's mean $\mu_{\mathrm{w}}$ for the $b_{1}^{*}, b_{2}^{*}$, and $b_{3}^{*}$ cases and at different qualities of the feature components. As a measurement of the feature quality we use the Gaussian channel capacity or entropy $H_{\mathrm{G}}$ as defined in [20]

$$
H_{\mathrm{G}}=\frac{1}{2} \log _{2}\left(1+\frac{\sigma_{\mathrm{b}}^{2}}{\sigma_{\mathrm{w}}^{2}}\right)
$$

which only depends on the ratio $\frac{\sigma_{\mathrm{b}}^{2}}{\sigma_{\mathrm{w}}^{2}}$ and where $\sigma_{\mathrm{b}}^{2}$ is the variance of the between-class Gaussian density $p_{\mathrm{b}}$ describing the variability of the mean $\mu_{\mathrm{w}}$ across the population and $\sigma_{\mathrm{w}}^{2}$ is the variance of the within-class Gaussian density $p_{\mathrm{w}}$.

Assuming the total density $p_{\mathrm{t}}$ to have a unit variance and using $\sigma_{\mathrm{t}}^{2}=\sigma_{\mathrm{w}}^{2}+\sigma_{\mathrm{b}}^{2}$ we can rewrite $H_{\mathrm{G}}$ as

$$
\begin{aligned}
H_{\mathrm{G}} & =\frac{1}{2} \log _{2}\left(1+\frac{\sigma_{\mathrm{t}}^{2}-\sigma_{\mathrm{w}}^{2}}{\sigma_{\mathrm{w}}^{2}}\right) \\
& =\frac{1}{2} \log _{2}\left(\frac{1}{\sigma_{\mathrm{w}}^{2}}\right) \\
& =-\log _{2}\left(\sigma_{\mathrm{w}}\right) .
\end{aligned}
$$

Hence, feature components with $H_{\mathrm{G}}=1$ have a within-class standard deviation of $\sigma_{\mathrm{w}}=\frac{1}{2^{H_{\mathrm{G}}}}=\frac{1}{2}$, similarly for the cases $H_{\mathrm{G}}=[2,3,4]$ we have $\sigma_{\mathrm{w}} \stackrel{2}{=}\left[\frac{1}{4}, \frac{1}{8}, \frac{1}{16}\right]$, respectively.

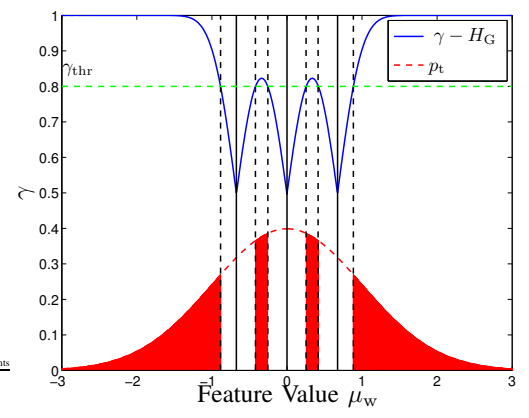

Fig. 9. The probability of selecting each quantization interval leading to a detection rate $\gamma$ larger than a threshold $\gamma_{\mathrm{thr}}$.

Using (1) the detection rate $\gamma$ for different values of $\mu_{\mathrm{w}}$ for different $b_{x}^{*}$ cases and feature qualities $H_{\mathrm{G}} \in\{1,2,3,4\}$ are shown in Fig. 8. Note that the quantization intervals are fixed because of the unit variance assumption of $p_{\mathrm{t}}$. The figures show that for the $b_{2}^{*}$ and $b_{3}^{*}$ cases the maximum detection rate $\gamma$ for the inner quantization intervals are much lower than for the outer intervals, because the width of the inner quantization intervals are much smaller in order to be equiprobable with respect to the total density. The detection rate difference between the inner and outer quantization bins depend on the feature quality $H_{\mathrm{G}}$ and on the $b_{x}^{*}$ case. A larger $\gamma$ difference is observed for smaller $H_{\mathrm{G}}$ values and when more bits are extracted.

As discussed in Section II, the DROBA algorithm maximizes the overall detection rate $\gamma_{t}$ as given by (2). Due to the optimization criteria, the DROBA algorithm tends to allocate multiple bits mostly for the cases where the subject's mean $\mu_{\mathrm{w}}$ is in the outer quantization intervals due to the larger $\gamma$ values. This behavior is stronger for the lower quality feature components because $\gamma$ is significantly larger for the outer quantization intervals as shown in Fig. 8.

We illustrate the non-uniformity effect introduced by the DROBA algorithm with the following simplified case. Consider the case where there are three feature components of equal quality of $H_{\mathrm{G}}=2$ from which four bits $\left(N_{\mathrm{B}}=4\right)$ have to be extracted and only two bits are allowed to be extracted from each component ( $b_{2}^{*}$ case). Assume, the first component analyzed has a detection rate of $\gamma_{1}=0.8$. The probability that the next component has a detection rate $\gamma_{2}$ larger than threshold $\gamma_{\mathrm{thr}}=\gamma_{1}$ is portrayed by the shaded area of the $p_{\mathrm{t}}$ density shown in Fig. 9 which is $\operatorname{Pr}\left(\gamma_{2}>\gamma_{\text {thr }}\right) \approx 0.5$. Note that the probability of each quantization interval is not equiprobable. For the outer quantization intervals we obtain $p\left(q_{1}\right)=p\left(q_{4}\right)=0.38$, while for the inner quantization intervals $p\left(q_{2}\right)=p\left(q_{3}\right)=0.12$. Hence the difference is $\delta_{2}=$ 0.26. If it turns out that $\gamma_{2}>\gamma_{1}$, then when analyzing the third component the threshold becomes $\gamma_{\mathrm{thr}}=\gamma_{2}$. Because of the larger $\gamma_{t h r}$ for the third component, the probability of obtaining a higher $\gamma_{2}$ in one of the quantization intervals becomes more uniform and $\delta_{2}$ is thus larger. Note that this effect is stronger for lower quality feature components with a smaller $H_{\mathrm{G}}$ or when more bits are extracted. 
